$\underline{\text { Review }}$

\title{
Use of antimicrobials in obstetric practice - A narrative review
}

\author{
M Patabendige ${ }^{1}$, RP Herath ${ }^{2}$, SR Athulathmudali ${ }^{3}$, WRPLI Wijesooriya ${ }^{4}$ \\ Sri Lankan Journal of Infectious Diseases 2020 Vol.10 (1):9-20 \\ DOI: http://dx.doi.org/10.4038/sljid.v10i1.8268
}

\begin{abstract}
Improvement of the outcome of infectious diseases by early and appropriate antimicrobial therapy is of paramount importance in any clinical discipline. Special considerations need to be given for the use of antimicrobial agents in obstetric practice, as it relates to the safety of both the mother and the fetus. However, their use and misuse are associated with significantly increasing antibiotic resistance. The objective of this review was to provide a useful overview for the practicing obstetrician, of current recommendations for the use of antibiotics in common situations related to pregnancy and the postpartum period.
\end{abstract}

Keywords: Antibiotic use; Obstetrics; Maternal health; Sepsis in pregnancy, Postpartum sepsis.

\section{Introduction}

Antibiotics account for $80 \%$ of all prescribed medications in obstetric practice. ${ }^{1}$ Only a few studies have examined the effects of antibiotics during human pregnancy on the fetus with resultant longterm and/or short-term sequelae. ${ }^{2}$

There are many guidelines and recommendations for the use of antimicrobials in obstetric care. Although most of the recommendations on the use of antimicrobials in obstetric practice are towards serious infections, there are many other indications for antimicrobial use during pregnancy. Guidelines and recommendations for antimicrobial use in such situations are often not available in a single accessible form. The objective of this review was therefore to provide a useful overview for the practicing clinician on current recommendations on the use of antibiotics in common situations related to pregnancy and the postpartum.

${ }^{1}$ Castle Street Hospital for Women, Colombo, Sri Lanka.

${ }^{2}$ Department of Obstetrics and Gynaecology, Faculty of Medicine, University of Kelaniya, Ragama, Sri Lanka

${ }^{3}$ Teaching Hospital- Mahamodara, Galle, Sri Lanka.

${ }^{4}$ Department of Microbiology, Faculty of Medicine, University of Kelaniya, Ragama, Sri Lanka

Address for correspondence: Dr Malitha Patabendige, Castle Street Hospital for Women, Colombo, Sri Lanka.

Telephone: +0094777357030 Email: mpatabendige@gmail.com

https://orcid.org/0000-0002-4092-7092

Received 15 October 2019 and revised version accepted 17 April 2020

(c) (i)

use, distribution, and reproduction in any medium, provided the original author and source are credited.

SLJID • www. http://sljol.info/index.php/SLJID • Vol. 10, No. 1, April 2020 


\section{Method}

In this narrative review, a comprehensive literature review was conducted relevant to the topic. This article presents the current recommended practice of antibiotic use in obstetric practice according to the available evidence to date. MEDLINE and Google scholar electronic databases were searched from January 2000 to June 2019, for English language original papers using predefined search terms such as antibiotic prophylaxis, antibiotic treatment, obstetric surgery, antibiotics in obstetrics and obstetric delivery. We also traced citations to and from relevant articles and searched our personal libraries for additional articles. As we were primarily interested in understanding the recommendations for antimicrobial use in current practice, current practice guidelines on this topic were also included.

Papers concerning the pharmacology of antibiotics during pregnancy and pathology and/or epidemiology of infections in pregnancy were excluded. Eligibility was determined by the first author.

\section{Results}

\section{Use of antimicrobial agents in the antenatal period}

\section{Urinary tract infections related to the pregnancy}

\section{Asymptomatic bacteriuria in pregnancy}

Asymptomatic bacteriuria occurs in 2\% to $10 \%$ of pregnancies and, if not treated, up to $30 \%$ of women will develop acute pyelonephritis. ${ }^{3}$ Asymptomatic bacteriuria has also been associated with low birth weight and preterm birth. ${ }^{3}$ It is therefore recommended to screen for bacteriuria during the first trimester with urine culture and if the culture is found to be positive, to treat according to the antibiotic sensitivity results. ${ }^{3,4}$ A 7-day course is recommended and prophylaxis is not recommended in a low-risk pregnancy. ${ }^{5}$

\section{Urinary tract infection in pregnancy}

Any medication used in pregnancy carries risks and benefits. However, untreated urinary tract infections are particularly associated with significant risk to the growing fetus such as spontaneous abortion, preterm birth, and low birth weight. ${ }^{6}$ Primary therapy is cephalexin $500 \mathrm{mg} 8$ hourly or nitrofurantoin $100 \mathrm{mg} 12$ hourly for 7 days according to the Sri Lankan guidelines of $2016 .{ }^{4}$ According to the NICE guidelines in the UK, amoxicillin, cephalexin or other antibiotics are recommended by the local microbiology team according to the culture and sensitivity pattern. ${ }^{5}$ Urine culture should be repeated after 48 hours of completion of antibiotics. ${ }^{3}$

Nitrofurantoin should be avoided in the third trimester because it is known to cause fetal haemolytic anemia. ${ }^{7}$ Co-amoxiclav should be avoided in less than 37 weeks of gestation due to increased risk of neonatal necrotizing enterocolitis. ${ }^{8}$

\section{Pyelonephritis in pregnancy}

Acute pyelonephritis is estimated to complicate up to $2 \%$ of all pregnancies. ${ }^{9}$ If left untreated, acute pyelonephritis could result in maternal morbidity including fever and acute renal failure and 
fetal complications including acute respiratory distress, stillbirth, and preterm birth. ${ }^{10}$ Urine culture and ABST with an ultrasonic assessment of renal tract would be essential supporting clinical findings in making a diagnosis of pyelonephritis.

According to the Sri Lankan guidelines of $2016^{4}$, first-line therapy is cefuroxime $750 \mathrm{mg}$ intravenous (IV) 8 hourly or co-amoxiclav 1.2g IV 8 hourly. Alternative treatment options may be needed in case of resistant organisms according to the culture and sensitivity pattern ${ }^{5}$ and these are ceftriaxone $1 \mathrm{~g}$ IV daily or cefotaxime $1 \mathrm{~g}$ IV 8 hourly. ${ }^{4}$ This IV therapy needs to be changed to oral therapy following clinical improvement and continued to complete 14 days (total duration) of antibiotics. $^{4}$

\section{Chickenpox in pregnancy}

Although it is an uncommon infection in pregnancy, varicella in pregnancy may carry a risk of higher morbidity, including pneumonia, hepatitis and encephalitis and rarely death. ${ }^{11}$ There are also fetal complications, especially if seroconversion has occurred in the first 28 weeks of pregnancy. $^{11}$

When there is a contact with chickenpox or shingles for a pregnant woman ${ }^{11}$,

a) A thorough history needs to be taken to confirm the significance of the potential contact and the susceptibility of the pregnant woman.

b) Those with an uncertain or no previous history of chickenpox or who come from susceptible territories and who have a significant exposure need to undertake a blood test to determine VZV immunity. When there is a significant exposure in a non-immune mother, she should be offered varicella-zoster immunoglobulin (VZIG) as early as possible.

c) VZIG is effective when given up to 10 days after contact. In the case of continuous exposures, this is 10 days from the appearance of the rash in the index case. In non-immune women, a significant exposure should be managed as potentially infectious from 8-28 days after exposure if they receive VZIG and from 8-21 days after exposure if they do not receive VZIG.

d) A second dose of VZIG may be needed if a further significant exposure has occurred and three weeks have gone from the last VZIG dose.

\section{Chickenpox infection}

In the case of established chickenpox infection with typical rash, oral aciclovir 800mg 5 times a day for 7 days should be started within the first 24 hours of the onset of the rash. ${ }^{411}$ Women with severe chickenpox are candidates for intravenous aciclovir. ${ }^{11}$ Aciclovir is not licensed for use in pregnancy and the potential risks and benefits should be discussed with the mother. ${ }^{11}$ However, rising evidence shows that there is no additional risk of major fetal anomalies with the exposure to aciclovir in pregnancy. ${ }^{12,13,14}$ Once chickenpox has developed with a typical rash, VZIG has no therapeutic benefit. ${ }^{11}$ 


\section{Respiratory tract infections in pregnancy}

Bacterial/viral pneumonia is difficult to differentiate, and perhaps viral pneumonia may have a bacterial co-infection. ${ }^{15}$ Empirical antibiotic treatment to cover common treatable bacterial pathogens is therefore usually recommended for the management of pneumonia. ${ }^{15}$

The initial choice of empirical antimicrobial treatment consists of a beta-lactam/cephalosporin and a macrolide together. ${ }^{16,17}$ Amoxicillin or amoxicillin-clavulanate are preferred beta-lactam antibiotics. The possible alternative antibiotics are ceftriaxone and cefuroxime. ${ }^{16,17}$ Often, there will be dramatic clinical improvement within 48 to 72 hours and the ongoing regimen resulting in improvement should not be altered within the first 72 hours. ${ }^{17}$ If there is a suspected aspiration, anaerobic cover is necessary. ${ }^{17}$

Possibility of infection with multi-drug resistant organisms causing pneumonia including penicillin-resistant Streptococcus pneumoniae and post-viral methicillin-resistant Staphylococcus aureus (MRSA) pneumonia are less common. ${ }^{15}$ However, aggressive therapy is needed in such situations. ${ }^{15}$ For MRSA pneumonia, intravenous vancomycin $(15 \mathrm{mg} / \mathrm{kg}$ every 12 hours, adjusted based on serum vancomycin levels) or linezolid (600 mg every 12 hours) is recommended. ${ }^{18}$

\section{Influenza in pregnancy}

Pregnant women are at increased risk of developing complications due to influenza, including spontaneous miscarriage, preterm birth, premature rupture of membranes, neonatal complications, secondary bacterial pneumonia in mother and even maternal death. ${ }^{4,19}$

Throat swabs should be sent for viral studies before starting antivirals and oral oseltamivir 75$150 \mathrm{mg} 12$ hourly should be given for 5 days. ${ }^{4}$ With regards to viral pneumonia, primary influenza pneumonia does not respond well to antiviral therapy (oseltamivir) and mortality remains high. ${ }^{17}$ Fortunately, severe primary influenza pneumonia is uncommon. ${ }^{17}$ Superimposed bacterial pneumonia should be treated with the appropriate antibiotics as given above. ${ }^{15,17}$

\section{Genital herpes in pregnancy}

Herpes infection can be potentially harmful to the neonate rather than the mother. ${ }^{20}$ Neonatal herpes can cause severe morbidity, including localized disease to the eye, skin, and mouth, encephalitis and disseminated multi-organ involvement. ${ }^{20}$ Maternal transmission of this rare, lethal infection must, therefore, be promptly managed. ${ }^{20}$

\section{First or second-trimester acquisition (until $27+6$ weeks of gestation)}

Management of the woman should be in line with her clinical condition and will usually involve the use of oral aciclovir in standard doses, $400 \mathrm{mg}$ three times daily, usually for 5 days. ${ }^{20}$ Intravenous aciclovir has to be considered for disseminated herpes simplex virus (HSV) infection. ${ }^{20}$ Following first or second trimester acquisition, daily suppressive aciclovir $400 \mathrm{mg}$ three times daily from 36 weeks of gestation reduces HSV lesions at term and hence the need for caesarean delivery. ${ }^{20,21,22}$ 


\section{Third-trimester acquisition (from 28 weeks of gestation)}

Oral aciclovir in standard dosage of $400 \mathrm{mg}$ three times daily for 5 days is the usual regime, unless for a severe infection that needs intravenous administration. ${ }^{20}$ After 28 weeks, therapy has to be continued with standard oral aciclovir as above until delivery. Caesarean delivery should be the preferred delivery method for all mothers with the first episode of genital herpes after 28 weeks. This is particularly important to consider in mothers with symptoms within 6 weeks of expected delivery as the risk of neonatal transmission (41\%) of HSV is very high in this period. ${ }^{20}$

\section{Women with recurrent genital herpes}

Women with recurrent genital herpes should be informed that the risk of neonatal herpes is low (0-3\% for vaginal delivery) even if lesions are present at the time of delivery. ${ }^{20}$ Daily suppressive aciclovir $400 \mathrm{mg}$ three times daily should be considered from 36 weeks of gestation although there is insufficient evidence to determine whether this reduces the incidence of neonatal herpes. ${ }^{20}$ However, it reduces viral shedding and recurrences at delivery so may reduce the need for caesarean delivery. ${ }^{20,23}$

\section{Spontaneous rupture of membranes}

\section{Preterm prelabour rupture of membranes (PPROM)}

PPROM complicates only $2 \%$ of pregnancies but is associated with $40 \%$ of preterm deliveries and can result in significant neonatal morbidity and mortality. ${ }^{24}$ The aim of treatment is to prevent preterm birth with its known complications as well as chorioamnionitis. ${ }^{24}$ Primary therapy should be oral erythromycin $250 \mathrm{mg} 6$ hourly for 10 days. ${ }^{24,25}$ A seven-day course of therapy with a combination of intravenous ampicillin and erythromycin followed by oral amoxicillin and erythromycin during expectant management can be also be considered as an alternative treatment option. ${ }^{26}$

\section{Prelabour rupture of membranes at or over 37 weeks of gestation (PROM)}

PROM at term (37-42 weeks) occurs in $8-10 \%$ of term pregnancies. ${ }^{27,28}$ Currently there is no convincing evidence of benefit to mothers or neonates for the routine use of antibiotics for PROM at or near term and therefore antibiotics are not recommended. ${ }^{25,28}$ These women should be offered induction of labour 24 hours after PROM or else depending on the local unit protocol. ${ }^{27,} 28,29$ Women with proven Group B Streptococcus (GBS) colonization including bacteriuria or a history of an infected baby need to have intrapartum antibiotic prophylaxis (IAP) as described below. ${ }^{4,30}$

\section{Intrapartum prophylaxis for Group B $\beta$ haemolytic streptococcus (GBS)}

Indications for IAP for GBS have been summarized below. ${ }^{4,30}$

\section{Indications for intrapartum GBS prophylaxis}
a. GBS carrier status
b. GBS bacteriuria in current pregnancy
c. Previous GBS infected baby (early- or late-onset)
d. Maternal pyrexia in labour $\left(\geq 38.2{ }^{\circ} \mathrm{C} / 100.8{ }^{\circ} \mathrm{F}\right)$ - therapeutic indication for broad- spectrum antibiotics including GBS cover
e. Rupture of membranes more than 18 hours
f. Confirmed preterm labour irrespective of the GBS maternal status 
First-line prophylaxis is benzylpenicillin $3 \mathrm{~g}(5 \mathrm{MU})$ IV loading dose followed by $1.5 \mathrm{~g}$ IV 4 hourly until delivery. ${ }^{40}$ Alternative prophylaxis options are ampicillin $2 \mathrm{~g}$ IV loading dose followed by $1 \mathrm{~g}$ IV 4 hourly until delivery. Alternatives include clindamycin $900 \mathrm{mg}$ IV 8 hourly or vancomycin 1g IV 12 hourly until delivery, especially in case of penicillin hypersensitivity. ${ }^{4}$ To optimize the efficacy of intrapartum antibiotic prophylaxis, the first dose should be given at least 2 hours before delivery. ${ }^{31,32}$

Maternal pyrexia in labour can indicate chorioamnionitis and a broad-spectrum antibiotic, rather than penicillin $G$, is recommended in this situation. ${ }^{30,33}$ The antibiotic regimen of choice will depend on local microbiology guidance. Intravenous amoxicillin $2 \mathrm{~g}$ every 6 hours (or intravenous cefuroxime $1.5 \mathrm{~g}$ every 6 hours in women with a non-anaphylactic reaction to penicillin) is acceptable in this situation. ${ }^{30,33}$

\section{Chorioamnionitis}

Chorioamnionitis or intra-amniotic infection is an acute inflammation of the membranes and chorion of the placenta, typically due to ascending polymicrobial bacterial infection in the setting of membrane rupture. ${ }^{34}$ Prompt initiation of antibiotic therapy is essential to prevent both maternal and fetal complications in the setting of clinical chorioamnionitis. ${ }^{35}$ Intrapartum antibiotic therapy should be considered for women with fever $>38.2^{\circ} \mathrm{C} / 100.8^{\circ} \mathrm{F}$ during labour. ${ }^{4}$ Necessary obstetric interventions should be carried out together with antibiotic therapy.

Primary therapy is a combination of ampicillin $2 \mathrm{~g}$ IV 6 hourly, gentamicin $5-7 \mathrm{mg} / \mathrm{kg}$ IV once daily and metronidazole 500mg IV 8 hourly until delivery. ${ }^{4,25}$ The American College of Obstetricians and Gynaecologists recommend only ampicillin and gentamicin as primary therapy and additional metronidazole only in post-caesarean patients. ${ }^{35}$ If cesarean delivery is performed, clindamycin (or metronidazole) every 8 hours is often added for anaerobic cover. ${ }^{34,35}$ The other option is to use ceftriaxone $2 \mathrm{~g}$ IV once daily and metronidazole 500mg IV 8 hourly. ${ }^{4}$ Alternatively, the combination of clindamycin $600 \mathrm{mg}$ IV 8 hourly with gentamicin $5-7 \mathrm{mg} / \mathrm{kg}$ IV once daily can be given. $^{4}$ If sexually transmitted infections (STI) are suspected, it is recommended to add azithromycin $1 \mathrm{~g}$ stat dose.

\section{Prophylaxis against infective endocarditis}

The current dogma of 'antibiotics for all' needs to be reconsidered, particularly as the widespread use of antibiotics promotes the emergence of resistant microorganisms, including resistant enterococci. ${ }^{36}$ However, because of the serious consequences and high costs associated with IE and the comparatively low costs associated with antibiotic prophylaxis, it has been demonstrated that antibiotic prophylaxis is likely to be very cost-effective (and even cost-saving) in preventing IE, particularly for those at high risk, even when the number of prevented IE cases is very low. ${ }^{37}$

The incidence of infective endocarditis (IE) in patients with cardiac disease is $0-1.2 \%$ and is higher in women with a mechanical prosthetic valve. ${ }^{36}$ The risk of IE would likely be influenced by the severity of valvular disease. ${ }^{36}$ Cardiac conditions for which IE prophylaxis is indicated include acquired valvular heart disease with stenosis or regurgitation, hypertrophic cardiomyopathy, 
previous infective endocarditis, structural congenital heart disease, including surgically corrected or palliated structural conditions valve replacement and rheumatic heart disease. ${ }^{36,37,38}$

IE prophylaxis is no longer recommended for conditions such as patients with mitral valve prolapse. ${ }^{36}$ Isolated atrial septal defect, fully repaired ventricular septal defect or fully repaired patent ductus arteriosus, and closure devices that are judged to be endothelialized have been excluded from prophylaxis. ${ }^{36,37,38}$ Prophylaxis for patients with the above indications should be considered for operative vaginal delivery, emergency or elective caesarean section, repair of third or fourth-degree perineal tear, manual removal of placenta and postpartum dilatation and curettage. Prophylaxis for IE is not recommended for women who are undergoing uncomplicated vaginal delivery, cervical cerclage and hysterectomy. ${ }^{36}$

The recommended regimen for prophylaxis is ampicillin $2 \mathrm{~g}$ IV as a single dose. ${ }^{4}$ Parenteral ceftriaxone $1 \mathrm{~g}$ (IV/intramuscular) or clindamycin $600 \mathrm{mg}$ IV as a single dose could be used for patients who are allergic to ampicillin. ${ }^{4}$ The timing of prophylactic antibiotics is 15 to 60 minutes prior to skin incision for caesarean deliveries and ideally at the time of membrane rupture for vaginal deliveries. ${ }^{4}$

\section{Caesarean birth}

The single most important risk factor for postpartum maternal infection is cesarean birth. Caesarean birth is a clean, contaminated surgery. Surgical antibiotic prophylaxis should be given for all women who are undergoing caesarean birth. ${ }^{4,40,41}$ Surgical antibiotic prophylaxis should generally be limited to a single dose. ${ }^{4,25,40,42}$

Cochrane review has recommended that prophylactic antibiotics should be routinely administered to all women undergoing cesarean section to prevent infection and this reduces the incidence of wound infection, endometritis and serious infectious complications by $60 \%$ to $70 \%{ }^{42}$ Studies have shown that single-dose antibiotic prophylaxis is as effective as multiple doses of antibiotic. ${ }^{41}$ Prophylactic antibiotics should ideally be given 30 minutes prior to the skin incision such as at the time of IV cannulation. ${ }^{4,39,40,42}$

There is evidence to support the use of surgical prophylaxis after clamping of the umbilical cord to prevent postpartum infections after caesarean. ${ }^{25,39}$ As a result, administration of antibiotics can still be done after delivery of the baby without jeopardizing the neonatal outcomes in a case of emergency caesarean birth. ${ }^{25}$ The Australian guidelines recommend antibiotics to be administered 30 -minutes prior to skin incision at the time of cannulation to be an effective bactericidal agent. ${ }^{39}$

For a mother with a significant history of anaphylaxis to other antibiotics or uncertain drug allergy, surgical prophylaxis can still be given immediate postpartum. ${ }^{40}$ Clinicians should be prepared with an emergency delivery protocol in a rare event of anaphylaxis in the pregnant mother. ${ }^{39}$

Narrow-spectrum antibiotics that are effective against Gram-positive and Gram-negative bacteria with some anaerobic coverage are the most appropriate choice. ${ }^{39}$ Primary therapy is cefuroxime $1.5 \mathrm{~g}$ IV single dose. ${ }^{4,25,39}$ For those who are allergic to cephalosporins, IV clindamycin along with gentamicin is an accepted alternative regime according to the Australian guidelines. ${ }^{39}$ The World 
Health Organization (WHO) recommends avoidance of co-amoxiclav in preterm births due to the high risk of neonatal necrotizing enterocolitis and emphasizes that it might be safer to avoid its use for caesarean birth of term babies too. ${ }^{25}$ Surgical prophylaxis should still be administered to mothers for whom caesarean birth is indicated who are on antibiotic prophylaxis for prolonged rupture of membranes or GBS prophylaxis. ${ }^{25,40}$

Vaginal cleansing with povidone-iodine immediately before caesarean birth is a recommended prophylactic measure against postpartum endometritis. ${ }^{25}$

\section{Other obstetric procedures}

Prophylaxis should be given for the repair of third or fourth-degree perineal tear. ${ }^{25,40,42}$ Third and fourth-degree tears involve the anal sphincter and thus give rise to a 'contaminated' or 'cleancontaminated' wound. First-line therapy is IV cephalosporin and metronidazole. ${ }^{25,40}$ This is in line with the recommendations of WHO and the Royal College of Obstetricians and Gynaecologists. ${ }^{18,25}$ However, the Australian guidelines do not support the use of antibiotic prophylaxis to prevent perineal wound complications following third or fourth-degree tears due to lack of robust evidence. ${ }^{39}$ However, they emphasize the need to make the decision depending on the individual case and clinical circumstances ${ }^{39}$ and this should be borne in mind when making decisions regarding antibiotics in all of these situations.

According to most clinical guidelines, antibiotic prophylaxis is not recommended for operative vaginal deliveries. ${ }^{25,39,43,44}$ The latest Cochrane review has concluded that evidence from a single trial suggests that antibiotic prophylaxis may lead to little or no difference in endometritis or maternal length of stay. ${ }^{44}$ However, good standards of hygiene and aseptic techniques are recommended. ${ }^{43}$ Available evidence also does not support the use of prophylactic antibiotics to reduce infectious morbidity following operative vaginal delivery, postpartum surgical evacuation of retained products and cervical cerclage and manual removal of placenta. ${ }^{39,42}$

Antibiotic prophylaxis is recommended by the WHO before manual removal of the placenta as a single dose of ampicillin or first-generation cephalosporin..$^{25,45}$

While there is inadequate randomized evidence to dictate uniform antibiotic prescribing practice in the above situations, the decision regarding prophylactic antibiotics should be made in each case, based on the clinical situation and individual patient circumstances such as length of labour, intrapartum fever, co-morbidities and extent of genital tract trauma, if any. ${ }^{40,46}$

Additional doses of prophylactic antibiotics may need to be given if an open abdominal surgical procedure is lasting more than three hours and/or estimated blood loss is greater than $1.5 \mathrm{~L}$ with a three to four hour gap from the initial antibiotic dose. ${ }^{42}$ 


\section{Use of antimicrobial agents in the postpartum period}

\section{Surgical site infections after caesarean birth}

Mild to moderate infections can be treated with oral cloxacillin 500mg 6 hourly or co-amoxiclav $625 \mathrm{mg} 8$ hourly. ${ }^{4}$ In severe infections, cefuroxime $750 \mathrm{mg}$ IV 8 hourly can be given with/without metronidazole $500 \mathrm{mg}$ IV 8 hourly or else co-amoxiclav $1.2 \mathrm{~g}$ IV 8 hourly. ${ }^{4}$ If the patient is allergic to penicillin, clindamycin oral $300 \mathrm{mg} 8$ hourly or IV $600 \mathrm{mg} 8$ hourly can be considered. ${ }^{4}$

\section{Episiotomy infections}

\section{Superficial infections}

These can be treated with cefuroxime 500mg 12 hourly with/without metronidazole 400mg 8 hourly or else a combination of clindamycin 300mg 8 hourly and gentamicin $5-7 \mathrm{mg} / \mathrm{kg}$ IV once daily. ${ }^{4}$

\section{Deep necrotizing fasciitis/myonecrosis}

Primary therapy is piperacillin-tazobactam $4.5 \mathrm{~g}$ IV 8 hourly or else a combination of ticarcillin clavulanate $3.2 \mathrm{~g}$ IV 8 hourly and clindamycin $600 \mathrm{mg}$ IV 8 hourly. ${ }^{4}$ Alternative treatment option is a combination of ceftriaxone $2 \mathrm{~g}$ IV daily and clindamycin $600 \mathrm{mg}$ IV 8 hourly. ${ }^{4}$

\section{Postpartum mastitis}

Primary therapy is oral cloxacillin 500mg 6 hourly. ${ }^{4}$ Severe infection needs cloxacillin $1 \mathrm{~g}$ IV 6 hourly. ${ }^{4}$ Alternative treatment option is cephalexin $500 \mathrm{mg} 8$ hourly. ${ }^{4}$ Clindamycin can be considered in patients with penicillin allergy. ${ }^{4}$ Ciprofloxacin/ fusidic acid are best avoided in breastfeeding mothers. ${ }^{4}$ Surgical intervention must be done where necessary in case of a breast abscess. $^{4}$

\section{Postpartum endometritis}

A combination of clindamycin and gentamicin is recommended as first-line antibiotics for the treatment of postpartum endometritis. ${ }^{25}$ Clindamycin can be administered as $600 \mathrm{mg}$ IV every six to eight hours, and gentamicin as $1-1.5 \mathrm{mg} / \mathrm{kg}$ or $60-80 \mathrm{mg}$ IV or IM every eight hours. ${ }^{25}$ Alternative therapy is a combination of ampicillin $2 \mathrm{~g}$ IV 6 hourly with gentamicin $5 \mathrm{mg} / \mathrm{kg}$ IV once daily and metronidazole 500mg IV 8 hourly. ${ }^{4,25}$ Other treatment options are a combination of coamoxiclav $1.2 \mathrm{~g}$ IV 8 hourly with gentamicin $5-7 \mathrm{mg} / \mathrm{kg}$ IV once daily or else combination of ceftriaxone $2 \mathrm{~g}$ IV daily with metronidazole $500 \mathrm{mg}$ IV 8 hourly. ${ }^{4}$ Treatment with antibiotics need to be continued for at least 24-48 hours after complete resolution of clinical manifestations including fever, uterine tenderness, purulent lochia, and/or leucocytosis. ${ }^{25}$ If cost and availability is a limiting factor, use of a penicillin class of drug (with gentamicin) is an acceptable $\mathrm{n}$ alternative for postpartum endometritis. ${ }^{25}$ 


\section{Conclusion}

This paper provides a comprehensive summary of currently recommended practice in the choice of antimicrobial agents in obstetric practice. However, due to the lack of robust scientific data, antimicrobial recommendations for some procedures are difficult to make. Therefore, clinicians should use clinical circumstances and case by case evaluation to decide rationally the need for antimicrobials. Regular audits to evaluate adherence to accepted recommendations in prescribing antibiotics may be helpful to determine the usefulness of guidelines. They would also assist in recognizing the need to change recommendations due to the development of resistance in commonly encountered pathogens. Observational and analytical studies and/or randomized controlled trials on the use of antibiotics in obstetric procedures where there is a lack of robust data are recommended.

\section{Conflicts of interest and financial disclosure}

There are no conflicts of interest and no relevant sources of funding.

\section{References}

1. Kuperman AA, Koren O. Antibiotic use during pregnancy: how bad is it? BMC Med. 2016; 17:14(1):91. doi: 10.1186/s12916-016-0636-0.

2. Bookstaver PB et al. A review of antibiotic use in pregnancy. Pharmacotherapy. 2015; 35(11):105262. doi:10.1002/phar.1649.

3. Smaill FM, Vazquez JC. Antibiotics for asymptomatic bacteriuria in pregnancy. Cochrane Database of Systematic Reviews 2019, Issue 11. Art. No.: CD000490.

4. Sri Lanka College of Microbiologists. Empirical and prophylactic use of antimicrobials: National Guidelines 2016. http://slmicrobiology.net/antibiotic-guidelines-2016/. Accessed 04 Feb 2020.

5. National Institute for Health and Clinical Excellence. Urinary tract infection (lower): antimicrobial prescribing. NICE guideline [NG109]: 2018. https://www.nice.org.uk/guidance/ng109/chapter/Summary-of-the-evidence. Accessed 04-04-2020.

6. Lamont HF, Blogg HJ, Lamont RF. Safety of antimicrobial treatment during pregnancy: a current review of resistance, immunomodulation and teratogenicity. Expert Opin Drug Saf. 2014; 13(12):1569-1581. doi:10.1517/14740338.2014.939580.

7. Cimolai N, Cimolai T. Nitrofurantoin and pregnancy. Canadian Medical Association Journal. 2007: 19; 176(13): 1860-1861. doi: https://dx.doi.org/10.1503\%2Fcmaj.1070028.

8. Kenyon S, Boulvain M, Neilson JP. Antibiotics for preterm rupture of membranes. Cochrane Database Syst Rev 2010; 8:CD 00058. doi: 10.1002/14651858.CD001058

9. Gilstrap LC, Ramin SM. Urinary tract infections during pregnancy. Obstetrics and Gynaecology Clinics North America. 2001;28(3):581-91. doi: 10.1016/S0889-8545(05)70219-9.

10. Farkash $\mathrm{E}$ et al. Acute antepartum pyelonephritis in pregnancy: a critical analysis of risk factors and outcomes. Eur J Obstet Gynecol Reprod Biol 2012; 162:24-7. doi: 10.1016/j.ejogrb.2012.01.024.

11. Royal College of Obstetricians and Gynaecologists. Chickenpox in pregnancy. Green-top Guideline No. 13; 2015. https://www.rcog.org.uk/en/guidelines-research-services/guidelines/gtg13/ Accessed 01-02-2020.

12. Stone KM, et al. Pregnancy outcomes following systemic prenatal acyclovir exposure: Conclusions from the international acyclovir pregnancy registry, 1984-99. Birth Defects Res A Clin Mol Teratol 2004;70: 201-7. 43. doi: 10.1002/bdra.20013.

13. Pasternak B, Hviid A. Use of acyclovir, valacyclovir and famciclovir in the first trimester of pregnancy and the risk of birth defects. JAMA 2010; 304:859-66. doi: 10.1001/jama.2010.1206. 
14. Mills JL, Carter TC. Acyclovir exposure and birth defects: an important advance, but more are needed. JAMA 2010; 304:905-6. doi: 10.1001/jama.2010.1214.

15. Metlay JP, Waterer GW, Long AC, et al. Diagnosis and treatment of adults with community-acquired pneumonia. An official clinical practice guideline of the American Thoracic Society and Infectious Diseases Society of America. Am J Respir Crit Care Med. 2019; 200(7):e45-e67. doi:10.1164/rccm.201908-1581 $1^{\text {ST }}$.

16. Mandell LA, et al. Infectious Diseases Society of America/American Thoracic Society Consensus Guidelines on the management of community-acquired pneumonia in adults. Clin Infect Dis. 2007; 44(suppl):S27-72. doi: 10.1086/511159.

17. Sheffield JS, Cunningham FG. Community-acquired pneumonia in pregnancy. Obstetrics and Gynaecology 2009; 114(4):915-922 doi: 10.1097/AOG.0b013e3181b8e76d.

18. Kalil AC, Metersky ML, Klompas M, et al. Management of adults with hospital-acquired and ventilator-associated pneumonia: 2016 Clinical Practice Guidelines by the Infectious Diseases Society of America and the American Thoracic Society. Clin Infect Dis. 2017 May 1;64(9):1298.

doi:10.1093/cid/ciw353.

19. Carlson A, Thung SF, Norwitz ER. H1N1 Influenza in pregnancy: What all obstetric care providers ought to know. Rev Obstet Gynecol. 2009; 2(3):139-45. PMID: 19826571; PMCID: PMC2760905.

20. British Association for Sexual Health and HIV (BASHH) and Royal College of Obstetricians and Gynaecologists. Management of genital herpes in pregnancy. 2014: 8-13.

https://www.rcog.org.uk/en/guidelines-research-services/guidelines/genital-herpes/. Accessed 01-022020.

21. Sheffield JS, et al. Acyclovir prophylaxis to prevent herpes simplex virus recurrence at delivery: a systematic review. Obstet Gynecol 2003; 102:1396-403.

doi: https://doi.org/10.1016/j.obstetgynecol.2003.08.015.

22. Brocklehurst $P$, et al. A randomised placebo controlled trial of suppressive acyclovir in late pregnancy in women with recurrent genital herpes infection. Br J Obstet Gynaecol 1998; 105:275-80. doi: 10.1111/j.1471-0528.1998.tb10086.x.

23. Gupta R, et al. Valacyclovir and acyclovir for suppression of shedding of herpes simplex virus in the genital tract. J Infect Dis. 2004; 190(8):1374-81. doi: 10.1086/424519.

24. Royal College of Obstetricians and Gynaecologists. Care of women presenting with suspected preterm prelabour rupture of membranes from 24+0 weeks of gestation. Green-top Guideline 2019; No.73. doi: https://doi.org/10.1111/1471-0528.15803.

25. World Health Organization. WHO recommendations for prevention and treatment of maternal peripartum infections. World Health Organization 2015.

http://www.who.int/reproductivehealth/publications/maternal_perinatal_health/peripartuminfections-guidelines/en/. Accessed 05-02-2020.

26. American College of Obstetricians and Gynecologists. Premature rupture of membranes. Practice Bulletin No. 188. Obstet Gynecol. 2018. 131(6).1163-1164. doi: 10.1097/AOG.0000000000002455.

27. National Institute for Health and Clinical Excellence. Inducing labour. NICE, Clinical guideline 70. 2008. https://www.nice.org.uk/Guidance/CG70. Accessed 05-02-2020.

28. Wojcieszek AM, Stock O.M, Flenady V. 2014 Antibiotics for prelabour rupture of membranes at or near term, Cochrane Database Systematic Reviews. 2014. CD001807. doi: 10.1002/14651858.CD001807.pub2

29. National Institute for Health and Clinical Excellence. Intrapartum care: Care of healthy women and their babies during childbirth. NICE Clinical Guideline 190. 2014. Last Updated 2017. https://www.nice.org.uk/guidance/cg190 Accessed 05-02-2020.

30. Hughes RG, et al on behalf of the Royal College of Obstetricians and Gynaecologists. Prevention of early-onset neonatal group B streptococcal disease. Green-top Guideline No. 36. BJOG 2017; 124:e280-e305. doi: https://doi.org/10.1111/1471-0528.14821. 
31. Lin FY, et al. The effectiveness of risk-based intrapartum chemoprophylaxis for the prevention of earlyonset neonatal group B streptococcal disease. Am J Obstet Gynecol. 2001; 184:1204-10.

doi: https://doi.org/10.1067/mob.2001.113875.

32. de Cueto M, et al. Timing of intrapartum ampicillin and prevention of vertical transmission of group B streptococcus. Obstet Gynecol 1998; 91:112-4.

doi: https://doi.org/10.1016/S0029-7844(97)00578-4.

33. Higgins RD, et al. Chorioamnionitis Workshop Participants. Evaluation and management of women and newborns with a maternal diagnosis of chorioamnionitis: summary of a workshop. Obstet Gynecol. 2016; 127:426-36. doi: 10.1097/AOG.0000000000001246.

34. Tita ATN, Andrews WW. Diagnosis and management of clinical chorioamnionitis. Clin Perinatol. 2010; 37(2): 339-354. doi: 10.1016/j.clp.2010.02.003.

35. Committee on Obstetric Practice. Committee Opinion No. 712: Intrapartum management of intraamniotic infection. Obstet Gynecol. 2017;1 30(2):e95-e101. doi:10.1097/AOG.0000000000002236.

36. Wilson W, Taubert KA, Gewitz M, et al. Prevention of infective endocarditis: A guideline from the American Heart Association Rheumatic Fever, Endocarditis, and Kawasaki Disease Committee, Council on Cardiovascular Disease in the Young, and the Council on Clinical Cardiology, Council on Cardiovascular Surgery and Anesthesia, and the Quality of Care and Outcomes Research

Interdisciplinary Working Group. Circulation 2008; 116:1736-54 doi: 10.1161/CIRCULATIONAHA.106.183095.

37. National Institute for Health and Clinical Excellence. Prophylaxis against infective endocarditis. Antimicrobial prophylaxis against infective endocarditis in adults and children undergoing interventional procedures. London, UK: NICE, Clinical guideline [CG 64]. 2008. Updated 2018. https://www.nice.org.uk/Guidance/cg64. Accessed 02-01-2020.

38. Tleyjeh IM, Steckelberg JM, Murad HS, et al. Temporal trends in infective endocarditis: a populationbased study in Olmsted County, Minnesota. JAMA 2005; 293(24):3022-8. doi: https://doi.org/10.1001/jama.293.24.3022.

39. The Royal Australian and New Zealand College of Obstetricians and Gynaecologists (RANZCOG). Prophylactic antibiotics in obstetrics and gynaecology: 2016. https://ranzcog.edu.au/RANZCOG_SITE/media/RANZCOGMEDIA/Women\%27s\%20Health/Statement\%20and\%20guidelines/Clinical\%20$\% 20 G e n e r a l / P r o p h y l a c t i c-a n t i b i o t i c s-i n-o b s t e t r i c s-a n d-g y n a e c o l o g y-(C-G e n-17)-R e v i e w-J u l y-$ 2016.pdf? ext=.pdf. Accessed 20-12-2019.

40. Clifford V, Daley A. Antibiotic prophylaxis in obstetric and gynaecology procedures: A Review. Aust New Z J of Obstet and Gynae 2012; 52: 412-19. doi:10.1111/j.1479-828X.2012.01460.x.

41. Smaill FM, Grivell RM. Antibiotic prophylaxis versus no prophylaxis for preventing infection after cesarean section. Cochrane Database Syst Rev. 2014; 10:CD007482. doi: 10.1002/14651858.CD007482.pub3.

42. Schalkwyk J, Eyk N. SOGC Clinical Practice Guideline: Antibiotic prophylaxis in obstetric procedures. Journal of Obstetrics and Gynaecology Canada 2010; 32(9):878-884. doi: https://doi.org/10.1016/S1701-2163(16)34662-X.

43. Royal College of Obstetricians and Gynaecologists. Operative vaginal delivery. Green-top Guideline No. 26; 2011. https://www.rcog.org.uk/en/guidelines-research-services/guidelines/gtg26/. Accessed 03-11-2019.

44. Liabsuetrakul T, Choobun T, Peeyananjarassri K, Islam QM. Antibiotic prophylaxis for operative vaginal delivery. Cochrane Database Syst Rev. 2017; 8: CD004455. doi: 10.1002/14651858

45. World Health Organization (WHO). WHO recommendations for the prevention and treatment of postpartum haemorrhage. 2012. http://apps.who.int/iris/bitstream/10665/75411/1/9789241548502_eng.pdf. Accessed 1.2.20

46. National Institute for Health and Clinical Excellence. Preterm labour and birth. NICE guideline [NG 25]: 2015. Updated August 2019. https://www.nice.org.uk/Guidance/NG25. Accessed 04-01-2020. 